\title{
Closed-Form Analytical Drain Current Model Considering Energy Transport and Self-Heating for Short-Channel Fully-Depleted SOI NMOS Devices With Lightly-Doped Drain Structure Biased in Strong Inversion
}

\author{
Shih-Chia Lin and James B. Kuo, Fellow, IEEE
}

\begin{abstract}
This paper reports a closed-form analytical drain current model considering energy transport and self-heating for short-channel fully-depleted (FD) SOI NMOS devices with lightly-doped drain (LDD) structure. As verified by the twodimensional (2-D) simulation results, the analytical drain current model considering energy transport and self-heating provides an accurate prediction of the drain current behavior of the $0.25-\mu \mathrm{m}$ FD SOI NMOS device with and without an LDD structure. From the analytical model, with the LDD structure, the device has a smaller effective electron mobility at a low drain voltage, where lattice temperature is dominant, and a higher effective mobility at a high drain voltage, where electron temperature dominates, as compared to the non-LDD device.
\end{abstract}

Index Terms-CMOS, compact model, SOI, VLSI.

\section{INTRODUCTION}

$\mathbf{S}$ OI technology has been receiving a lot of attention owing to its potential for VLSI. As for bulk devices, high electric field effects in SOI MOS devices can cause reliability problems [1], [2]. Due to buried oxide isolation, high electric field effects of the SOI MOS devices may be quite different from those of the bulk devices. For SOI MOS devices with a short-channel length, high electric field effects such as impact ionization, parasitic BJT, and thermal effects have been discussed [3]-[6]. Recently, an electron/lattice temperature related mobility model that includes the energy balance equation has also been reported for short-channel SOI MOS devices [7], [8]. Lightly-doped drain (LDD) structures have been used in fully-depleted (FD) SOI MOS devices to reduce high electric field effects [9]-[13]. When the LDD structure is accounted for, closed-form analytical models are difficult to obtain. An analytical threshold voltage model considering the sidewall fringing electric field

Manuscript received May 20, 2002; revised September 6, 2002. This paper was supported under a research grant from NSERC in Canada. The review of this paper was arranged by Editor C. C. McAndrew.

S.-C. Lin is with the Department of Electrical and Computer Engineering, University of Waterloo, Waterloo, ON N2L 3G1, Canada (e-mail: jbkuo@ uwaterloo.ca)

J. B. Kuo is with the Department of Electrical and Computer Engineering, University of Waterloo, Waterloo, ON N2L 3G1, Canada, on leave from National Taiwan University, Taipei, Taiwan, R.O.C. (e-mail: j.kuo@ieee.org).

Digital Object Identifier 10.1109/TED.2002.805222 effect for the short-channel FD SOI MOS devices with LDD structure has been reported [14]. Until now, no closed-form analytical drain current models for short-channel FD SOI MOS devices with LDD structure considering the electron and lattice temperature-related mobility model are available. In order to facilitate SPICE circuit simulation, in this paper, a closed-form analytical drain current model considering energy transport and self-heating for short-channel FD SOI NMOS devices with LDD structure using energy balance equation is presented. Since electron and lattice temperature effects are more noticeable in the strong inversion regime [7], this paper concentrates on developing an analytical model for the device biased in strong inversion. In the following sections, derivation of the model is described first, followed by model verification, and discussion.

\section{MODEL Derivation}

Fig. 1 shows the cross section of the FD SOI NMOS device with LDD structure under study. As shown in the figure, in order to simplify the analysis, the LDD structure without an overlap with the gate has been adopted in the FD SOI NMOS device. In order to simplify the analysis, the gate overlap capacitance is not considered. In addition, the fringing electric field effect via the oxide sidewall spacer is neglected. The LDD region occupies the whole thin-film region [11]. In the following derivation, the thermal effect model is described first, followed by the electron temperature model using the energy balance equation, and the mobility model. Finally, the whole analytical drain current model for the FD SOI NMOS device with LDD structure biased in the saturation region is presented.

\section{A. Thermal Effect}

For an FD SOI NMOS device with the LDD structure, as shown in Fig. 1, the poor heat conduction capability of the buried oxide results in the heat dissipation, leading to a rise in the lattice temperature. The lattice temperature $\left(T_{l}\right)$ is related to power consumption $(P)$ of the device as follows:

$$
P R_{\mathrm{th}}=T_{l}-T_{0}
$$




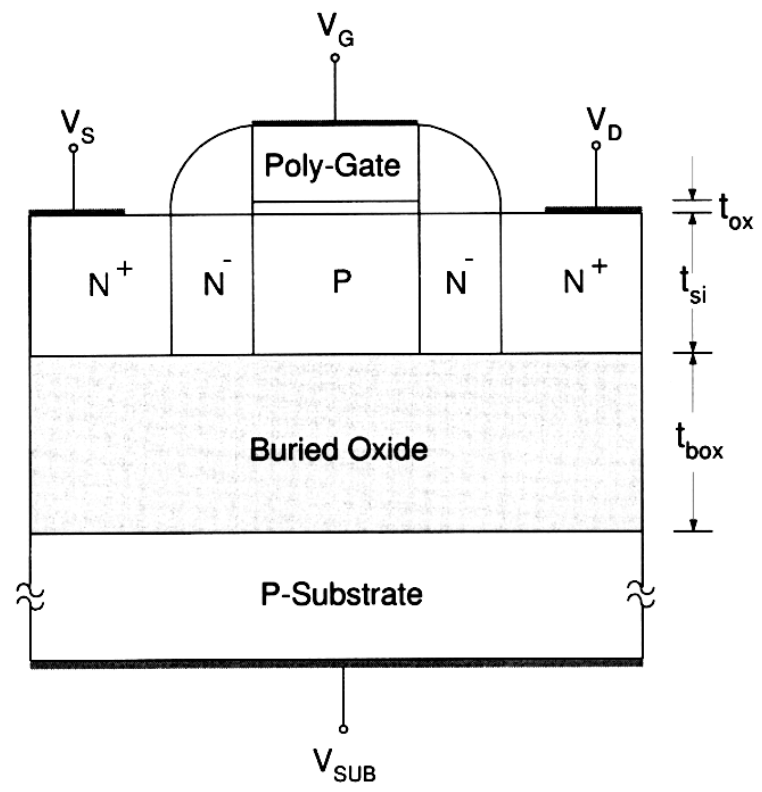

(a)

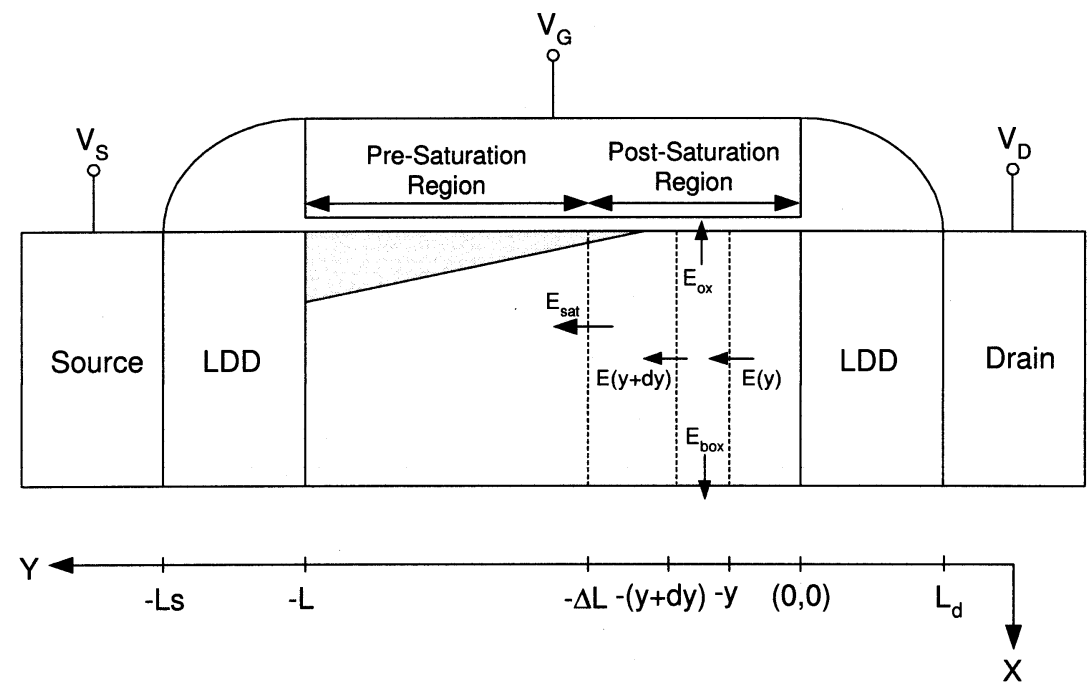

(b)

Fig. 1. Cross section of the (a) FD SOI NMOS device with (b) LDD structure under study.

where $T_{l}$ is the lattice temperature and power consumption $(P)$ is the product of the drain current $\left(I_{D}\right)$ and the drain voltage $\left(V_{D}\right): P=I_{D} V_{D} \cdot R_{\mathrm{th}}$ is the thermal resistance of the device: $R_{\text {th }}=(1) /(2 W)\left[\left(t_{\text {box }}\right) /\left(K_{\text {ox }} K_{d} t_{\text {si }}\right)\right]^{(1 / 2)}[7]$, where $W$ is the channel width, $t_{\mathrm{box}}$ is the thickness of the buried oxide, and $t_{\mathrm{si}}$ is the thickness of the thin film. $K_{\mathrm{ox}}$ and $K_{d}$ are the thermal conductivity of oxide and silicon, respectively. According to [7], for the device with a channel width of $9.5 \mu \mathrm{m}, K_{\mathrm{ox}}=1 \mathrm{~W} / \mathrm{m} \cdot \mathrm{K}$, $K_{d}=63 \mathrm{~W} / \mathrm{m} \cdot \mathrm{K}$. Thus, the thermal resistance of the device is $R_{\mathrm{th}}=1.48 \times 10^{4} \mathrm{~K} / \mathrm{W}$. (Note that the heat loss considered in (1) is mainly via the substrate. Heat loss may also be via metal contacts, which can be taken into account by adjusting the thermal resistance value $\left(R_{\mathrm{th}}\right)$. Using the model developed in this paper, when $R_{\mathrm{th}}$ is decreased, which implies consideration of the heat loss via the metal contact, the lattice temperature and the electron temperature become smaller.)

\section{B. Energy Transport}

From energy balance equation [7], the lattice temperature $\left(T_{l}\right)$, the electron temperature $\left(T_{n}\right)$, and the electric field $(E)$ in the lateral channel of the device are related to the power consumption as follows:

$$
\begin{aligned}
E^{2} & =\frac{3 k}{2 q} \frac{1+\frac{\alpha k}{q}\left(T_{n}-T_{l}\right)}{\mu_{s}\left(T_{l}\right)} \frac{T_{n}-T_{l}}{\tau_{\epsilon}}-\frac{5 k E}{2 q} \frac{d T_{n}}{d y} \\
\alpha & =\frac{3 \mu_{s}\left(T_{l}\right)}{2 v_{\mathrm{sat}}^{2} \tau_{\epsilon}}
\end{aligned}
$$

where $y$ is in the lateral channel direction, as shown in Fig. 1, and $k$ is the Boltzmann constant. Considering lattice temperature, the electron surface mobility $\left(\mu_{s}\left(T_{l}\right)\right)$ is expressed as [7]: $\mu_{s}\left(T_{l}\right)=\mu_{s 0}\left(T_{l} / T_{0}\right)^{-2}$, where $\mu_{s 0}=\left(\mu_{0}\right) /\left(1+\theta\left(V_{G}-V_{T}\right)\right)$ is the electron surface mobility at the ambient temperature $\left(T_{0}\right)$, 
and $\theta$ is surface mobility coefficient. Here, $\theta=0.3$ and $\mu_{0}=$ $400 \mathrm{~cm}^{2} / \mathrm{Vs}$ have been adopted [7]. $V_{G}$ is the gate voltage, $V_{T}$ is the threshold voltage of the device, $\tau_{\epsilon}$ is the energy relaxation time, and $v_{\text {sat }}$ is the electron saturation velocity.

For an FD SOI NMOS device, analysis of its drain current characteristics can be divided into two regions: 1) triode region $\left(V_{D}<V_{\mathrm{DSAT}}\right)$ and 2) saturation region $\left(V_{D}>V_{\mathrm{DSAT}}\right)$.

\section{Triode Region}

In this subsection, an analytical model for the FD SOI NMOS device with LDD structure, biased in the triode region is described. For the FD SOI NMOS device with LDD structure, before the drain current model can be organized, the mobility model based on the lattice temperature and the electron temperature, which can be derived from the energy balance equation, should be derived first. Here, from the two-dimensional (2-D) Poisson's equation, the electric field in the channel is derived first. Then, using the energy balance equation, the electron temperature, and thus the mobility model are derived.

a) Potential and electric field: In this FD SOI NMOS device under study, 2-D Poisson's equation has been used to obtain the potential distribution in the center channel and two $n$-LDD regions

$$
\frac{\partial^{2} V(x, y)}{\partial x^{2}}+\frac{\partial^{2} V(x, y)}{\partial y^{2}}=-\frac{q N}{\epsilon_{\mathrm{si}}}
$$

where

$x \quad$ vertical substrate direction;

$y \quad$ lateral channel direction;

$q$ electronic charge;

$\epsilon_{\mathrm{si}} \quad$ silicon permittivity;

$N$ doping density.

$N=N_{D}$ if n-type and $N=-N_{A}$ if p-type. The four boundary conditions [14] are

1) at the front gate interface, the surface potential is $V(0, y)=V_{\mathrm{sf}}(y)$

2) at the buried oxide interface, the surface potential is $V\left(t_{\mathrm{si}}, y\right)=V_{\mathrm{sb}}(y)$

$3)$ at the front gate interface, the electric field is related to the front gate voltage $\left(V_{G}\right)$ as $(\partial V(x, y)) /\left.(\partial x)\right|_{x=0}=$ $-\left(\epsilon_{\mathrm{ox}} / \epsilon_{\mathrm{si}}\right)\left(V_{G}-V_{\mathrm{sf}}(y)\right) /\left(t_{\mathrm{ox}}\right)$, where $\epsilon_{\mathrm{ox}}$ is oxide permittivity, $\epsilon_{\mathrm{si}}$ is silicon permittivity, and $t_{\mathrm{ox}}$ is the front gate oxide thickness;

4) at the buried oxide interface, the electric field is related to the back gate voltage $\left(V_{\mathrm{SUB}}\right)$ as: $(\partial V(x, y)) /\left.(\partial x)\right|_{x=t_{\mathrm{si}}}=-\left(\epsilon_{\mathrm{ox}} / \epsilon_{\mathrm{si}}\right)\left(V_{\mathrm{sb}}(y)-V_{\mathrm{SUB}}\right) /$ $\left(t_{\text {box }}\right)$.

In order to solve the 2-D Poisson's equation with the boundary conditions, a second-order polynomial in terms of the vertical substrate direction $(x)$ has been used to approximate the potential distribution in the center channel and two $n$-LDD regions

$$
V(x, y)=a_{0}(y)+a_{1}(y) x+a_{2}(y) x^{2}
$$

where $a_{0}(y), a_{1}(y)$, and $a_{2}(y)$ are functions of $y$. Considering that at the source $n$-LDD/channel and the channel/drain $n$-LDD boundaries, the electric field and the potential are continuous, based on the derivation from [14], the surface potential in the center channel region is expressed as (see Appendix for derivation)

$$
V_{\mathrm{sf}}(y)=A_{0} e^{r_{0}(y+L)}+B_{0} e^{-r_{0}(y+L)}-\frac{q N_{A} k_{s}}{r_{0}^{2} \epsilon_{\mathrm{si}}}-\frac{\beta_{1}}{r_{0}^{2}}
$$

where coefficients are expressed as

$$
\begin{aligned}
& A_{0}=\frac{-a_{12} V_{D}+W_{1}}{a_{11} a_{22}-a_{12} a_{21}} \\
& B_{0}=\frac{a_{11} V_{D}+W_{2}}{a_{11} a_{22}-a_{12} a_{21}} \\
& a_{11}=\cosh \left(r_{1} L_{s}\right)-\frac{r_{0}}{r_{1}} \sinh \left(r_{1} L_{s}\right) \\
& a_{12}=\cosh \left(r_{1} L_{s}\right)+\frac{r_{0}}{r_{1}} \sinh \left(r_{1} L_{s}\right) \\
& a_{21}=e^{r_{0} L}\left[\cosh \left(r_{1} L_{d}\right)+\frac{r_{0}}{r_{1}} \sinh \left(r_{1} L_{d}\right)\right] \\
& a_{22}=e^{-r_{0} L}\left[\cosh \left(r_{1} L_{d}\right)-\frac{r_{0}}{r_{1}} \sinh \left(r_{1} L_{d}\right)\right] \\
& W_{0}=\frac{-q N_{D} k_{s}}{r_{1}^{2} \epsilon_{\mathrm{si}}}+\frac{\beta_{2}}{r_{1}^{2}}-\frac{q N_{A} k_{s}}{r_{0}^{2} \epsilon_{\mathrm{si}}}-\frac{\beta_{1}}{r_{0}^{2}} \\
& W_{1}=\left[\frac{-q N_{D} k_{s}}{r_{1}^{2} \epsilon_{\mathrm{si}}}+\frac{\beta_{2}}{r_{1}^{2}}\right]\left(a_{22}-a_{12}\right) \\
& +W_{0}\left\{\cosh \left(r_{1} L_{s}\right) \cosh \left(r_{1} L_{d}\right)\left(1-e^{-r_{0} L}\right)\right. \\
& +\frac{r_{0}}{r_{1}}\left[\cosh \left(r_{1} L_{d}\right) \sinh \left(r_{1} L_{s}\right)\right. \\
& \left.\left.+e^{-r_{0} L} \sinh \left(r_{1} L_{d}\right) \cosh \left(r_{1} L_{s}\right)\right]\right\} \\
& W_{2}=\left[\frac{-q N_{D} k_{s}}{r_{1}^{2} \epsilon_{\mathrm{si}}}+\frac{\beta_{2}}{r_{1}^{2}}\right]\left(a_{11}-a_{21}\right) \\
& \text { - } W_{0}\left\{\cosh \left(r_{1} L_{d}\right) \cosh \left(r_{1} L_{s}\right)\left(1-e^{r_{0} L}\right)\right. \\
& -\frac{r_{0}}{r_{1}}\left[\cosh \left(r_{1} L_{d}\right) \sinh \left(r_{1} L_{s}\right)\right. \\
& \left.\left.+e^{r_{0} L} \sinh \left(r_{1} L_{d}\right) \cosh \left(r_{1} L_{s}\right)\right]\right\} \\
& r_{0}=\sqrt{\frac{2 k_{s}}{t_{\mathrm{si}} \epsilon_{\mathrm{si}}} \frac{C_{\mathrm{ox}} C_{\mathrm{box}}+C_{\mathrm{ox}} C_{s}+C_{\mathrm{box}} C_{s}}{2 C_{s}+C_{\mathrm{box}}}} \\
& r_{1}=\sqrt{\frac{2 k_{s}}{t_{\mathrm{si}}^{2}} \frac{C_{\mathrm{box}}}{2 C_{s}+C_{\mathrm{box}}}} \\
& \beta_{1}=-\frac{2 k_{s}}{t_{\mathrm{si}} \epsilon_{\mathrm{si}}} \frac{C_{o x}\left(C_{s}+C_{\mathrm{box}}\right) V_{G}+C_{s} C_{\mathrm{box}} V_{\mathrm{SUB}}}{2 C_{s}+C_{\mathrm{box}}} \\
& \beta_{2}=-\frac{2 k_{s}}{t_{\mathrm{si}}^{2}} \frac{C_{\mathrm{box}} V_{\mathrm{SUB}}}{2 C_{s}+C_{\mathrm{box}}} .
\end{aligned}
$$

In the above coefficient expressions, $N_{A}$ is the p-type doping density of the center channel region in the thin film and $N_{D}$ is the n-type doping density of the $n$-LDD regions near source and drain. $C_{\mathrm{box}}, C_{\mathrm{ox}}$, and $C_{s}$ are the unit-area capacitances of the buried oxide, the front gate oxide, and the thin film, respectively. $k_{s}\left(0<k_{s}<1\right)$ is a parameter to describe the relationship between the derivative of the lateral electric field in the silicon thin film and the derivative of the surface lateral electric field [15]: $\left(\partial^{2} V(x, y) / \partial y^{2}\right) \cong\left(1 / k_{s}\right)\left(d^{2} V_{\text {sf }}(y) / d y^{2}\right)$, where $V_{\text {sf }}(y)$ 
is the surface potential at the front gate oxide interface. $L_{s} / L_{d}$ is the width of the depletion region in the $n$-LDD region near source/drain in the thin film

$$
\begin{aligned}
& L_{s}=\sqrt{\frac{2 \epsilon_{\mathrm{si}}}{q} \frac{N_{A} V_{\mathrm{bi}}}{\left(N_{A}+N_{D}\right) N_{D}}} \\
& L_{d}=\sqrt{\frac{2 \epsilon_{\mathrm{si}}}{q} \frac{N_{A}\left(V_{\mathrm{bi}}+V_{D}\right)}{\left(N_{A}+N_{D}\right) N_{D}}}
\end{aligned}
$$

where

$V_{\mathrm{bi}}$ built-in voltage $\left(V_{\mathrm{bi}}=(k T / q) \ln \left(\left(N_{D} N_{A}\right) / n_{i}^{2}\right)\right)$;

$n_{i} \quad$ intrinsic concentration;

$T$ ambient temperature in Kelvin.

b) Electron temperature: Differentiating (5), the lateral electric field at the surface of the thin film $\left(E_{\mathrm{sf}}(y)\right)$ is

$$
E_{\mathrm{sf}}(y)=-A_{0} r_{0} e^{r_{0}(y+L)}+B_{0} r_{0} e^{-r_{0}(y+L)} .
$$

From the surface electric field equation [(7)] and solving the energy balance equation [(2)], the electron temperature profile in the device can be obtained. Since the electron temperature profile is strongly correlated to the surface electric field profile [(7)] in the channel, the electron temperature profile can be assumed to be

$$
T_{n}(y)=M_{0} e^{r_{0}(y+L)}+N_{0} e^{-r_{0}(y+L)}+R_{0} .
$$

From (2), (7), and (8), since the coefficients of the $e^{2 r_{0}(y+L)} /$ the $e^{-2 r_{0}(y+L)}$ terms on both sides of the equation should be equivalent, one obtains

$$
\begin{aligned}
& M_{0}=\frac{q r_{0} \mu_{s}\left(T_{l}\right) \tau_{\epsilon} A_{0}}{3 \alpha k}\left[-\frac{5 r_{0}}{2}+\sqrt{\frac{25 r_{0}^{2}}{4}+\frac{9}{v_{\text {sat }}^{2} \tau_{\epsilon}^{2}}}\right] \\
& N_{0}=\frac{q r_{0} \mu_{s}\left(T_{l}\right) \tau_{\epsilon} B_{0}}{3 \alpha k}\left[-\frac{5 r_{0}}{2}-\sqrt{\frac{25 r_{0}^{2}}{4}+\frac{9}{v_{\text {sat }}^{2} \tau_{\epsilon}^{2}}}\right]
\end{aligned}
$$

where $v_{\text {sat }}$ is the electron saturation velocity $\left(4 \times 10^{7} \mathrm{~cm} / \mathrm{s}\right)$, and $\tau_{\epsilon}$ is the energy relaxation time (0.03 ps). From (2), (7)-(9), considering the energy balance equation at the drain end in the channel $(y=0)$, one obtains $(10)$, shown at the bottom of the page. c) Electron mobility: The temperature-dependent electron mobility can be expressed as a function of the difference between the electron temperature and the lattice temperature [7]

$$
\mu_{n}=\frac{\mu_{s}\left(T_{l}\right)}{1+\frac{\alpha k}{q}\left(T_{n}-T_{l}\right)}
$$

From the above equation, the electron mobility is a function of the lateral channel. In order to simplify the analysis such that a closed-form analytical model can be obtained, an effective approach is adopted. The effective electron mobility is defined as $\mu_{\mathrm{eff}}=L / \int_{0}^{L}\left(1 / \mu_{n}\right) d y$, which is too complex for obtaining an analytical model. This effective electron mobility is approximated as the average of the values at the source $(y=-L)$ and the drain ends $(y=0)$ of the channel: $\mu_{\mathrm{eff}}=(2) /\left(\left(1 /\left(\mu_{n}(0)\right)+\left(1 / \mu_{n}(-L)\right)\right.\right.$. From (8) and (11), the electron mobility at the drain and the source ends are $\mu_{n}(0)=\left(\mu_{s}\left(T_{l}\right)\right) /\left(1+(\alpha k / q)\left[M_{0} e^{r_{0} L}+N_{0} e^{-r_{0} L}+R_{0}-\right.\right.$ $\left.\left.\left.T_{l}\right]\right) ; \mu_{n}(-L)=\left(\mu_{s}\left(T_{l}\right)\right) /\left(1+(\alpha k / q)\left[M_{0}+N_{0}+R_{0}-T_{l}\right]\right)\right)$. Therefore, the effective electron mobility becomes

$$
\begin{aligned}
\mu_{\mathrm{eff}}= & \frac{4 \mu_{s}\left(T_{l}\right)}{3+\sqrt{1+S \mu_{s}^{2}\left(T_{l}\right)}} \\
S= & 4 r_{0}^{2} \tau_{\epsilon}^{2}\left[\frac{A_{0}^{2} m^{2} e^{2 r_{0} L}+B_{0}^{2} n^{2} e^{-2 r_{0} L}}{9}-\frac{2 A_{0} B_{0}}{v_{\mathrm{sat}}^{2} \tau_{\epsilon}^{2}}\right. \\
& \left.-\frac{25 r_{0}^{2} A_{0} B_{0}}{9}\right] .
\end{aligned}
$$

Using Taylor's expansion formula to approximate (12), the effective electron mobility becomes

$$
\begin{aligned}
\mu_{\mathrm{eff}} & \cong A T_{l}^{2}+B T_{l}+C \\
A & =\left.\frac{1}{2} \frac{\partial^{2} \mu_{\mathrm{eff}}}{\partial T_{l}^{2}}\right|_{T_{l}=T_{0}} \\
B & =\left.\frac{\partial \mu_{\mathrm{eff}}}{\partial T_{l}}\right|_{T_{l}=T_{0}}-\left.\frac{\partial^{2} \mu_{\mathrm{eff}}}{\partial T_{l}^{2}}\right|_{T_{l}=T_{0}} T_{0} \\
C & =\left.\mu_{\mathrm{eff}}\right|_{T_{l}=T_{0}}-\left.\frac{\partial \mu_{\mathrm{eff}}}{\partial T_{l}}\right|_{T_{l}=T_{0}} T_{0}+\left.\frac{1}{2} \frac{\partial^{2} \mu_{\mathrm{eff}}}{\partial T_{l}^{2}}\right|_{T_{l}=T_{0}} T_{0}^{2} \cdot
\end{aligned}
$$

$$
\begin{aligned}
R_{0}= & T_{l}-\frac{q}{2 \alpha k}-\left(M_{0} e^{r_{0} L}+N_{0} e^{-r_{0} L}\right) \\
& +\frac{q}{2 \alpha k} \sqrt{1+4 r_{0}^{2} \tau_{\epsilon}^{2}\left[\frac{A_{0}^{2} m^{2} e^{2 r_{0} L}+B_{0}^{2} n^{2} e^{-2 r_{0} L}}{9}-\frac{2 A_{0} B_{0}}{v_{\mathrm{sat}}^{2} \tau_{\epsilon}^{2}}-\frac{25 r_{0}^{2} A_{0} B_{0}}{9}\right] \mu_{s}^{2}\left(T_{l}\right)} \\
m= & -\frac{5 r_{0}}{2}+\sqrt{\frac{25 r_{0}^{2}}{4}+\frac{9}{v_{\mathrm{sat}}^{2} \tau_{\epsilon}^{2}}} \\
n= & -\frac{5 r_{0}}{2}-\sqrt{\frac{25 r_{0}^{2}}{4}+\frac{9}{v_{\mathrm{sat}}^{2} \tau_{\epsilon}^{2}}} .
\end{aligned}
$$


In the triode region, with the effective electron mobility [(13)], the drain current is obtained [7]

$$
I_{D}=\mu_{\mathrm{eff}} C_{\mathrm{ox}} \frac{W}{L}\left[\left(V_{G}-V_{T}\right) V_{D}-a_{0} V_{D}^{2}\right] \frac{1}{1+\frac{V_{D}}{E_{\mathrm{sat}} L}}
$$

where drain-induced conductivity enhancement (DICE) has been included [16], [17]. Note that $E_{\mathrm{sat}}=\left(2 v_{\mathrm{sat}}\right) /\left(\mu_{0}\right)$ is the critical electric field when the traveling electron reaches the saturated velocity. In (14), $a_{0}=(1 / 2)\left(C_{s}+C_{\mathrm{ox}}\right) /\left(C_{\mathrm{ox}}\right)$ and $V_{T}$ is the threshold voltage. Considering drain-induced barrier lowering (DIBL) and temperature dependence [16], [17], the threshold voltage can be expressed as $V_{T}=V_{T 0}-\delta_{T}\left(T_{l}-T_{0}\right)-\delta_{D} V_{\mathrm{DS}}$, where $V_{T 0}$ is the threshold voltage without considering the temperature and DIBL effects. $\delta_{T}$ and $\delta_{D}$ are the coefficients to consider the temperature and DIBL effects, respectively. From (1), (13), and (14), the lattice temperature is obtained

$$
\begin{aligned}
T_{l}\left(V_{D}, V_{G}\right) \\
=\frac{-(B f-1)-\sqrt{(B f-1)^{2}-4 A f\left(C f+T_{0}\right)}}{2 A f} \\
f=C_{\mathrm{ox}} \frac{W}{L}\left[\left(V_{G}-V_{T 0}\right) V_{D}-a_{0} V_{D}^{2}\right] V_{D} R_{\mathrm{th}} \frac{1}{1+\frac{V_{D}}{E_{\mathrm{sat}} L}} .
\end{aligned}
$$

Note that in order to simplify the analysis, $\delta_{T}$ and $\delta_{D}$ have been neglected in $f$. From (13) and (15), the effective electron mobility is obtained. Then, using (14), the drain current of the FD SOI NMOS device with LDD structure biased in the triode region is obtained.

\section{Saturation Region}

For an FD SOI NMOS device biased in the saturation region $\left(V_{\mathrm{DS}}>V_{\mathrm{DSAT}}\right)$, the lateral channel region is divided into two regions: 1) pre-saturation region and 2) post-saturation region, separated by the boundary at $y=-\Delta L$, where the potential is $V_{\mathrm{DSAT}}$. Using a similar approach as reported in [7] and [18], at the boundary of pre-saturation/post-saturation, $V_{\mathrm{DSAT}}$ is defined as

$$
V_{\mathrm{DSAT}}=V_{\mathrm{DSAT} 1}+V_{p}-\left(V_{\mathrm{DSAT} 1}^{n}+V_{p}^{n}\right)^{\frac{1}{n}}
$$

where $V_{p}$ is the drain voltage of a conventional long-channel device, which is biased with pinchoff at the drain $\left(d I_{D} / d V_{D}\right)=$ $0)$ and $V_{\mathrm{DSAT1}}$ is the drain voltage when the velocity saturation occurs at drain. $n$ is used to smooth the transition between $V_{p}$ and $V_{\mathrm{DSAT} 1}$ for determining $V_{\mathrm{DSAT}} \cdot n=2$ is usually used. In order to simplify the calculation of $V_{p}$, Taylor's approximation has been used for the effective electron mobility equation [(12)] at the drain voltage of $V_{D}=\left(V_{G}-V_{T}\right) /\left(2 a_{0}\right)$. As a result

$$
\begin{aligned}
& \mu_{\mathrm{eff}}=\frac{4 \mu_{\mathrm{sp}}}{P_{0}+P_{1} V_{D}} \\
& P_{0}=3+\frac{S_{2}\left(\frac{V_{G}-V_{T}}{2 a_{0}}\right)+2 S_{3}}{2 \sqrt{S_{1}\left(\frac{V_{G}-V_{T}}{2 a_{0}}\right)^{2}+S_{2}\left(\frac{V_{G}-V_{T}}{2 a_{0}}\right)+S_{3}}} \\
& P_{1}=\frac{2 S_{1}\left(\frac{V_{G}-V_{T}}{2 a_{0}}\right)+S_{2}}{2 \sqrt{S_{1}\left(\frac{V_{G}-V_{T}}{2 a_{0}}\right)^{2}+S_{2}\left(\frac{V_{G}-V_{T}}{2 a_{0}}\right)+S_{3}}} \\
& S_{1}=\frac{4 r_{0}^{2} \tau_{\epsilon}^{2} \mu_{\mathrm{sp}}^{2}}{\left(a_{11} a_{22}-a_{12} a_{21}\right)^{2}}\left[\frac{a_{12}^{2} m^{2} e^{2 r_{0} L}+a_{11}^{2} n^{2} e^{-2 r_{0} L}}{9}\right. \\
& \left.+a_{12} a_{11}\left(\frac{2}{v_{\mathrm{sat}}^{2} \tau_{\epsilon}^{2}}+\frac{25 r_{0}^{2}}{9}\right)\right] \\
& S_{2}=\frac{4 r_{0}^{2} \tau_{\epsilon}^{2} \mu_{\mathrm{sp}}^{2}}{\left(a_{11} a_{22}-a_{12} a_{21}\right)^{2}} \\
& \times\left[\frac{-2 a_{12} W_{1} m^{2} e^{2 r_{0} L}+2 a_{11} W_{2} n^{2} e^{-2 r_{0} L}}{9}\right. \\
& \left.-\left(a_{11} W_{1}-a_{12} W_{2}\right)\left(\frac{2}{v_{\text {sat }}^{2} \tau_{\epsilon}^{2}}+\frac{25 r_{0}^{2}}{9}\right)\right] \\
& S_{3}=1+\frac{4 r_{0}^{2} \tau_{\epsilon}^{2} \mu_{\mathrm{sp}}^{2}}{\left(a_{11} a_{22}-a_{12} a_{21}\right)^{2}} \\
& \times\left[\frac{W_{1}^{2} m^{2} e^{2 r_{0} L}+W_{2}^{2} n^{2} e^{-2 r_{0} L}}{9}\right. \\
& \left.-W_{1} W_{2}\left(\frac{2}{v_{\text {sat }}^{2} \tau_{\epsilon}^{2}}+\frac{25 r_{0}^{2}}{9}\right)\right] \\
& \mu_{\mathrm{sp}}=\mu_{s 0}\left(\frac{T_{0}}{T_{l}\left(\frac{V_{G}-V_{T}}{2 a_{0}}, V_{G}\right)}\right)^{2} .
\end{aligned}
$$

From (14) and (17), with the condition that $\left(d I_{D} / d V_{D}\right)=0$, $V_{p}$ can be obtained as

$$
V_{p}=\frac{-a_{0} P_{0}+\sqrt{\left(a_{0} P_{0}\right)^{2}+\left(V_{G}-V_{T}\right) a_{0} P_{0} P_{1}}}{a_{0} P_{1}} .
$$

At the drain voltage of $V_{\mathrm{DSAT} 1}$, at which the carrier electrons reach the saturated velocity, the product of the electron mobility at drain $\left(\mu_{n}(0)\right)$ and the electric field $(E(0))$ is related to the saturated velocity as

$$
-\mu_{n}(0) E(0)=\eta_{2} v_{\text {sat }}
$$

where $\eta_{2}$ is a fitting parameter [7]. Here, $\eta_{2}=1$. Therefore, from (7) and (19), one obtains (20), shown at the bottom of the next page. From (16), the potential $V_{\mathrm{DSAT}}$, at which the oper-

$$
\begin{aligned}
V_{\mathrm{DSAT} 1} & =\frac{-\left(2 Q_{1} Q_{2}-S_{2}\right)+\sqrt{\left(2 Q_{1} Q_{2}-S_{2}\right)^{2}-4\left(Q_{2}^{2}-S_{1}\right)\left(Q_{1}^{2}-S_{3}\right)}}{2\left(Q_{2}^{2}-S_{1}\right)} \\
Q_{1} & =-1-\frac{2 \mu_{\mathrm{sp}}}{\eta_{2} v_{\mathrm{sat}}} \frac{W_{2} e^{-r_{0} L}-W_{1} e^{r_{0} L}}{a_{11} a_{22}-a_{12} a_{21}} r_{0} \\
Q_{2} & =-\frac{2 \mu_{\mathrm{sp}}}{\eta_{2} v_{\mathrm{sat}}} \frac{a_{11} e^{-r_{0} L}+a_{12} e^{r_{0} L}}{a_{11} a_{22}-a_{12} a_{21}} r_{0} .
\end{aligned}
$$


ation of the device enters from the triode region into the saturation region, is equal to the minimum of $V_{p}$ and $V_{\mathrm{DSAT} 1}$. This implies that in the vicinity of the triode/saturation boundary, it is determined by either the channel reach pinchoff or the carrier electrons reaching the saturated velocity-the mechanism in the device occurs first dominates the triode/saturation transition. From (16) to (20), $V_{\mathrm{DSAT}}$ is a function of the drain voltage, the lattice temperature, and the electron temperature.

For the device operating in the saturation region, the channel can be divided into the pre-saturation region and the post-saturation region.

1) Pre-Saturation Region: In the pre-saturation region, the drain current formula still assumes the triode expression [(14)] except that the channel length is replaced by $L-\Delta L$, where $\Delta L$ is the length of the post-saturation region.

2) Post-Saturation Region: For an FD SOI NMOS device with LDD structure, its post-saturation region is adjacent to the drain $n$-LDD region. Applying 2-D Gauss law to the post-saturation region and the depletion region in the $n$-LDD region, and using the boundary conditions that at the boundary the potential and electric field are continuous, the width of the post-saturation region can be obtained.

a) Potential and electric field: As shown in Fig. 1(b), select a Gauss box in the post-saturation region between $y$ and $y+d y$. Applying 2-D Gauss law around the Gauss box with the $x$ direction electric field at the top and the bottom as: $E_{\mathrm{ox}}=$ $\left(V_{G}-V_{\mathrm{sf}}(y)\right) /\left(t_{\mathrm{ox}}\right), E_{\mathrm{box}}=\left(V_{\mathrm{SUB}}-V_{\mathrm{sb}}(y)\right) /\left(t_{\mathrm{box}}\right)$ and the $y$ direction electric field at right and left as: $E(y), E(y+d y)$, one obtains

$$
\begin{array}{r}
\epsilon_{\mathrm{si}} t_{\mathrm{si}} \frac{d E(y)}{d y}+\epsilon_{\mathrm{ox}}\left[\frac{V_{G}-V_{\mathrm{sf}}(y)}{t_{\mathrm{ox}}}+\frac{V_{\mathrm{SUB}}-V_{\mathrm{sb}}(y)}{t_{\mathrm{box}}}\right] \\
=-q t_{\mathrm{si}} N+Q_{m}
\end{array}
$$

where $N=-N_{A}$ in the channel region and $N=N_{D}$ in the $n$-LDD region. $Q_{m}$ is the mobile charge density.

From (4) with the boundary conditions and [14], in the channel region, the front surface potential $\left(V_{\mathrm{sf}}(y)\right)$ and the back surface potential $\left(V_{\mathrm{sb}}(y)\right)$ are related as

$$
\begin{aligned}
V_{\mathrm{sb}}(y)=\frac{2 C_{s}+C_{\mathrm{ox}}}{2 C_{s}+C_{\mathrm{box}}} V_{\mathrm{sf}}(y)- & \frac{C_{\mathrm{ox}}}{2 C_{s}+C_{\mathrm{box}}} V_{G} \\
& +\frac{C_{\mathrm{box}}}{2 C_{s}+C_{\mathrm{box}}} V_{\mathrm{SUB}} .
\end{aligned}
$$

In the $n$-LDD region, in order to simplify calculation, the fringing electric field due to the oxide sidewall spacer is neglected- $(\partial V(x, y)) /\left.(\partial x)\right|_{x=0}=0$ [14]. Hence, from (4) with the boundary conditions and [14], the front surface potential and the back surface potential are related as

$$
V_{\mathrm{sb}}(y)=\frac{2 C_{s}}{2 C_{s}+C_{\mathrm{box}}} V_{\mathrm{sf}}(y)+\frac{C_{\mathrm{box}}}{2 C_{s}+C_{\mathrm{box}}} V_{\mathrm{SUB}} .
$$

As for the mobile charge density $Q_{m}$ in (21), considering at $y=-\Delta L$, the channel switches from pre-saturation to post-saturation with the potential $V_{\mathrm{sf}}(-\Delta L)=V_{\mathrm{DSAT}}$. Since in the presaturation region the gradient of the laterial electric field is small and can be neglected, thus one can assumes that at the boundary of the pre-saturation/post-saturation region $(d E(y)) /(d y)=0$ [19], thus the mobile charge density becomes

$$
Q_{m}=\epsilon_{\mathrm{ox}}\left(\frac{V_{G}-V_{\mathrm{sf}}(-\Delta L)}{t_{\mathrm{ox}}}+\frac{V_{\mathrm{SUB}}-V_{\mathrm{sb}}(-\Delta L)}{t_{\mathrm{box}}}\right)
$$

In the channel region, from (21), (22), and (24), a second-order differential equation in terms of the surface channel potential is obtained

$$
\begin{aligned}
\frac{d^{2} V_{\mathrm{sf}}(y)}{d y^{2}} & =\frac{V_{\mathrm{sf}}(y)-V_{\mathrm{DSAT}}}{l^{2}} \\
l & =\sqrt{\frac{\epsilon_{\mathrm{si}} t_{\mathrm{si}}}{2} \frac{2 C_{s}+C_{\mathrm{box}}}{C_{s} C_{\mathrm{box}}+C_{\mathrm{ox}} C_{\mathrm{box}}+C_{s} C_{\mathrm{ox}}}} .
\end{aligned}
$$

Considering the boundary conditions: $V_{\mathrm{sf}}(-\Delta L)=V_{\mathrm{DSAT}}$, $E_{\mathrm{sf}}(-\Delta L)=E_{\text {sat }}$, solving the differential equation, one obtains the surface channel potential and the surface electric field as

$$
\begin{aligned}
& V_{\mathrm{sf}}(y)=l E_{\mathrm{sat}} \sinh \left(\frac{y+\Delta L}{l}\right)+V_{\mathrm{DSAT}} \\
& E_{\mathrm{sf}}(y)=E_{\mathrm{sat}} \cosh \left(\frac{y+\Delta L}{l}\right) .
\end{aligned}
$$

In the $n$-LDD region, since the fringing electric field effect is not considered, the $\left(V_{G}-V_{\mathrm{sf}}(y)\right) /\left(t_{\mathrm{ox}}\right)$ term in (21) is neglected. With (21), (23), and (24), a second-order differential equation is obtained

$$
\begin{aligned}
\frac{d^{2} V_{\mathrm{sf}}(y)}{d y^{2}} & =\frac{V_{\mathrm{sf}}(y)-V_{\mathrm{DSAT}}}{l_{1}^{2}}-\frac{q\left(N_{D}-N_{A}\right)}{\epsilon_{\mathrm{si}}} \\
l_{1} & =\sqrt{\frac{\epsilon_{\mathrm{si}} t_{\mathrm{si}}}{2} \frac{2 C_{s}+C_{\mathrm{box}}}{C_{s} C_{\mathrm{box}}}} .
\end{aligned}
$$

Since at the channel $/ n$-LDD boundary, the electric field and the potential are continuous, from (26) $-V_{\mathrm{sf}}(0)=V_{\mathrm{DSAT}}+$ $l E_{\mathrm{sat}} \sinh (\Delta L / l), E_{\mathrm{sf}}(0)=E_{\mathrm{sat}} \cosh (\Delta L / l)$, and (27), one obtains

$$
\begin{aligned}
V_{\mathrm{sf}}(y)= & l E_{\mathrm{sat}} \sinh \left(\frac{\Delta L}{l}\right) \cosh \left(\frac{y}{l_{1}}\right) \\
& +l_{1} E_{\mathrm{sat}} \cosh \left(\frac{\Delta L}{l}\right) \sinh \left(\frac{y}{l_{1}}\right)+V_{\mathrm{DSAT}} \\
& +\left[1-\cosh \left(\frac{y}{l_{1}}\right)\right] \frac{q\left(N_{D}-N_{A}\right) l_{1}^{2}}{\epsilon_{\mathrm{si}}} \\
E_{\mathrm{sf}}(y)= & \frac{l}{l_{1}} E_{\mathrm{sat}} \sinh \left(\frac{\Delta L}{l}\right) \sinh \left(\frac{y}{l_{1}}\right) \\
& +E_{\mathrm{sat}} \cosh \left(\frac{\Delta L}{l}\right) \cosh \left(\frac{y}{l_{1}}\right) \\
& -\frac{q\left(N_{D}-N_{A}\right) l_{1}}{\epsilon_{\mathrm{si}}} \cdot \sinh \left(\frac{y}{l_{1}}\right)
\end{aligned}
$$


At the $n$-LDD/drain boundary $\left(y=L_{d}\right) V_{\mathrm{sf}}\left(L_{d}\right)=V_{D}$, from (28), the length of the post-saturation region $(\Delta L)$ is obtained

$$
\begin{aligned}
\Delta L & =l \sinh ^{-1}\left[\frac{-G_{1} G_{3}+G_{2} \sqrt{G_{1}^{2}+G_{3}^{2}-G_{2}^{2}}}{G_{1}^{2}-G_{2}^{2}}\right] \\
G_{1} & =l E_{\mathrm{sat}} \cosh \left(\frac{L_{d}}{l_{1}}\right) \\
G_{2} & =l_{1} E_{\mathrm{sat}} \sinh \left(\frac{L_{d}}{l_{1}}\right) \\
G_{3} & =\left[1-\cosh \left(\frac{L_{d}}{l_{1}}\right)\right] \frac{q\left(N_{D}-N_{A}\right) l_{1}^{2}}{\epsilon_{\mathrm{si}}}+V_{\mathrm{DSAT}}-V_{D} .
\end{aligned}
$$

From (29) and (16), $\Delta L$ is a function of $V_{D}$ and $T_{l}$.

b) Impact ionization: In the saturation region, due to the high electric field in the channel near drain, impact ionization is important. Thus, in the drain current model, impact ionization effect should be included [8]

$$
\begin{aligned}
I_{D}= & G \cdot I_{\mathrm{ch}}+H \cdot I_{\mathrm{CB} 0} \\
G= & 1+\frac{(M-1)\left(1-(1-K) \alpha_{0}\right)}{1-\left(1+K \cdot K^{\prime}(M-1)\right) \alpha_{0}} \\
H= & \frac{1+K^{\prime}(M-1)}{1-\left(1+K \cdot K^{\prime}(M-1)\right) \alpha_{0}} \\
I_{\mathrm{CB} 0}= & W t_{\mathrm{si}} \frac{I_{s 0}}{1+\theta_{I}\left(V_{G}-V_{T}\right)} \\
M= & 1+\frac{\gamma \lambda^{2}}{B_{0}\left(\lambda+\lambda_{0}\right)}\left(V_{D}-V_{\mathrm{DSAT}}\right) \\
& \times \exp \left[-\frac{B_{0}\left(\lambda+\lambda_{0}\right)}{V_{D}-V_{\mathrm{DSAT}}}\right]
\end{aligned}
$$

where $I_{\text {ch }}$ is the drain current as shown in (14). While considering the saturation region, $L$ should be replaced by $L-\Delta L$ and $V_{D}$ should be replaced by $V_{\mathrm{DSAT}} . I_{s 0}$ is the leakage current, which is used to account for the collector-base leakage current $\left(I_{\mathrm{CB} 0}\right)$ in the parasitic BJT with the emitter-base open in the FD SOI NMOS device. From [8], $I_{s 0}=10 \mathrm{~A} / \mathrm{cm}^{2} . M$ is the multiplication factor while considering impact ionization. $K$ and $K^{\prime}$ are used to account for the mechanism of the impact ionization hole current and the collector current in the parasitic BJT [8]. $\alpha_{0}$ is the current gain of the parasitic BJT. When in saturation, the lattice temperature can be obtained by (1), (13), (14), (30), and (31), shown at the bottom of the page. From the lattice temperature [(31)], $V_{\mathrm{DSAT}}$ can be obtained using (16), (18), and (20). Then, using the effective mobility [(17)] and $V_{\mathrm{DSAT}}[(16)]$ and from (30), the drain current formula considering impact ionization of the FD SOI NMOS device with LDD structure biased in the saturation region can be obtained. Note that lattice temperature vaires as a function of position along the channel. In order to simplify the analysis, the variation of the lattice temperature along the channel has been neglected - an average lattice temperature approach has been used.

\section{E. Without LDD Structure}

The previous derivation in Sections II-C and II-D is for the FD SOI NMOS device with LDD structure. For FD SOI NMOS device without LDD structure (non-LDD structure) - the $n$-LDD region is replaced by the $\mathrm{n}^{+}$source/drain region, the derivation of the surface channel potential $V_{\mathrm{sf}}(y)$ from (3) and (4) only in the channel region is sufficient. Therefore, without LDD structure, the surface channel potential is similar to (5)

$$
V_{\mathrm{sf}}(y)=A_{0} e^{r_{0}(y+L)}+B_{0} e^{-r_{0}(y+L)}-\frac{q N_{A} k_{s}}{r_{0}^{2} \epsilon_{\mathrm{si}}}-\frac{\beta_{1}}{r_{0}^{2}}
$$

except that $A_{0}$ and $B_{0}$ are modified as follows:

$$
\begin{aligned}
& A_{0}=\frac{1}{2 \sinh \left(r_{0} L\right)} V_{D}+\frac{\left(1-e^{-r_{0} L}\right)}{2 \sinh \left(r_{0} L\right)}\left(\frac{q N_{A} k_{s}}{r_{0}^{2} \epsilon_{\mathrm{si}}}+\frac{\beta_{1}}{r_{0}^{2}}\right) \\
& B_{0}=-\frac{1}{2 \sinh \left(r_{0} L\right)} V_{D}+\frac{\left(e^{r_{0} L}-1\right)}{2 \sinh \left(r_{0} L\right)}\left(\frac{q N_{A} k_{s}}{r_{0}^{2} \epsilon_{\mathrm{si}}}+\frac{\beta_{1}}{r_{0}^{2}}\right) .
\end{aligned}
$$

Without LDD structure, the drain current model equation described before is still applicable except that the following coefficients need to be replaced:

$$
\begin{aligned}
a_{11} & =-1 \\
a_{12} & =-1, \\
a_{11} a_{22}-a_{12} a_{21} & =2 \sinh \left(r_{0} L\right) \\
W_{1} & =\left(1-e^{-r_{0} L}\right)\left(\frac{q N_{A} k_{s}}{r_{0}^{2} \epsilon_{\mathrm{si}}}+\frac{\beta_{1}}{r_{0}^{2}}\right) \\
W_{2} & =\left(e^{r_{0} L}-1\right)\left(\frac{q N_{A} k_{s}}{r_{0}^{2} \epsilon_{\mathrm{si}}}+\frac{\beta_{1}}{r_{0}^{2}}\right) .
\end{aligned}
$$

\section{Model Evaluation ANd Discussion}

In order to assess the effectiveness of the analytical model for the FD SOI NMOS device with LDD structure, the analytical model results have been compared with the results using a 2-D simulator [20] and experimental data [11]. Fig. 1 shows the cross section of the FD SOI NMOS device with LDD structure under study. The FD SOI NMOS device has a front gate oxide of $70 \AA$, a thin film of $800 \AA$ doped with a p-type density of $10^{17} \mathrm{~cm}^{-3}$, a buried oxide of $4000 \AA$, with a channel length of $0.25 \mu \mathrm{m}$, a channel width of $9.5 \mu \mathrm{m}$, and an LDD

$$
\begin{aligned}
T_{l}\left(V_{D}, V_{G}\right) & =\frac{-\left(G B f^{\prime}-1\right)-\sqrt{\left(G B f^{\prime}-1\right)^{2}-4 G A f^{\prime}\left(G C f^{\prime}+H I_{C B 0} V_{D} R_{\mathrm{th}}+T_{0}\right)}}{2 G A f^{\prime}} \\
f^{\prime} & =C_{\mathrm{ox}} \frac{W}{L-\Delta L}\left[\left(V_{G}-V_{T 0}\right) V_{\mathrm{DSAT}}-a_{0} V_{\mathrm{DSAT}}^{2}\right] V_{D} R_{\mathrm{th}} \frac{1}{1+\frac{V_{D}}{E_{\mathrm{sat}} L}} .
\end{aligned}
$$




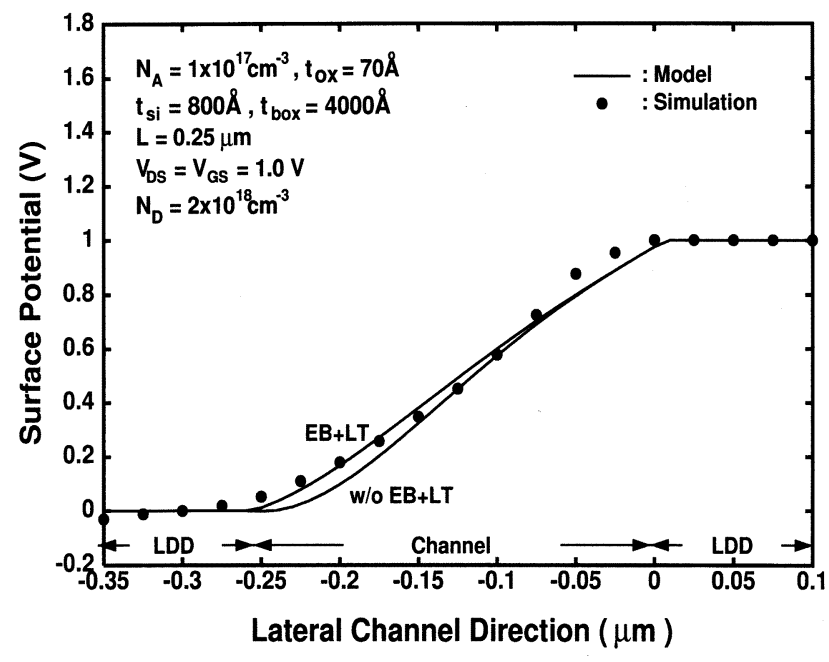

Fig. 2. Potential distribution in the lateral surface channel of the FD SOI NMOS device with a front gate oxide of $70 \AA$, a thin film of $800 \AA$ doped with a p-type density of $10^{17} \mathrm{~cm}^{-3}$, a buried oxide of $4000 \AA$, with a channel length of $0.25 \mu \mathrm{m}$, an $n$-LDD structure of $0.1 \mu \mathrm{m}$ in length doped with an n-type density of $2 \times 10^{18} \mathrm{~cm}^{-3}$, biased at $V_{\mathrm{GS}}=1 \mathrm{~V}$ and $V_{\mathrm{DS}}=1 \mathrm{~V}$, based on the model result and the 2-D simulation result.

structure of $0.1 \mu \mathrm{m}$ in length doped with an n-type density of $2 \times 10^{18} \mathrm{~cm}^{-3}$. The thermal resistance of this device assumes the value as described in Section II-A. For the 2-D simulation of this test device, energy transport, lattice temperature, and impact ionization models have been included. In the analytical model, the impact ionization model [(30)] has been adopted with the following fitting parameter values obtained from simulation results- $K=K^{\prime}=0.95, \alpha_{0}=0.95, \theta_{I}=0.3, \gamma=4 \times 10^{10}$ $\mathrm{cm}^{-2}, B_{0}=6.5 \times 10^{5} \mathrm{~V} / \mathrm{cm}, \lambda_{0}=900 \AA$, and $\lambda=450 \AA$ [8]. The threshold voltage model has been adopted with the following parameter values $-\delta_{T}=0.002 / \mathrm{K}$ and $\delta_{D}=50 \mathrm{mV} / \mathrm{V}$ to account for the temperature dependence and the DIBL effects [16], [17].

Fig. 2 shows the potential distribution in the lateral surface channel of the FD SOI NMOS device with an $n$-LDD structure, biased at $V_{\mathrm{GS}}=1 \mathrm{~V}$ and $V_{\mathrm{DS}}=1 \mathrm{~V}$, based on the model result and the 2-D simulation result, with and without considering energy transport and self-heating. As shown in the figure, considering the energy transport and self-heating (EB+LT) the surface potential in the center channel region is slightly higher than that without considering the energy transport and self-heating (without EB+LT). As shown in the figure, the analytical model result considering energy transport and self-heating $(\mathrm{EB}+\mathrm{LT})$ - the upper solid line is closer to the 2-D simulation result considering EB and LT.

Fig. 3 shows drain current characteristics of the FD SOI NMOS device with an LDD structure based on the experimental data, the model and the 2-D simulation results. As shown in the figure, without considering self-heating (EB only), both the simulation (solid square) and the model (dashed line) results indicate an overestimated drain current as compared to the experimental data [11]. Considering both energy transport and self-heating $(\mathrm{EB}+\mathrm{LT})$, the analytical model (solid line) can accurately predict the drain current characteristics for the device with LDD structure as verified by the 2-D simulation results (solid circle) and the experimental data (empty circle).

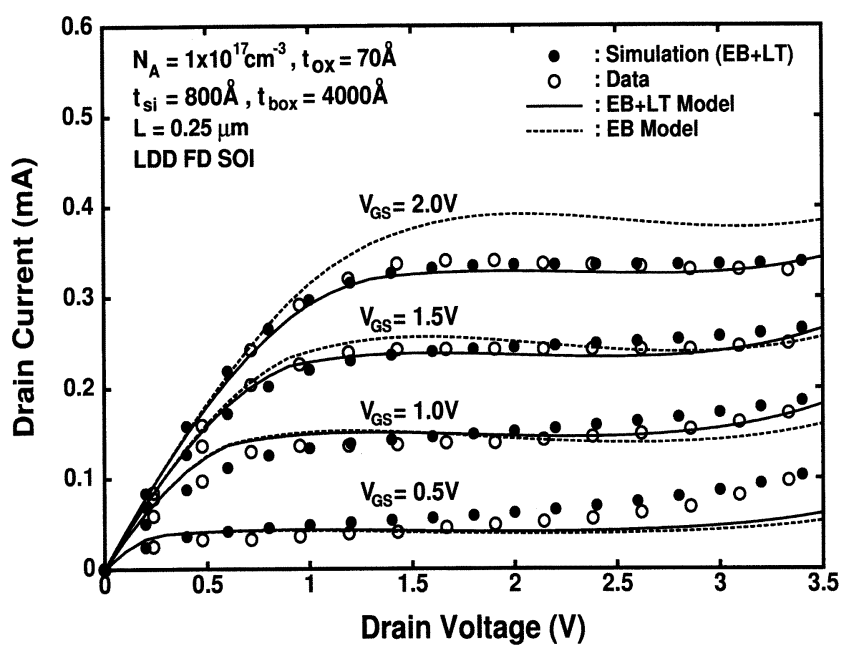

Fig. 3. Drain current characteristics of the FD SOI NMOS device with an LDD structure based on the experimental data, the model, and the 2-D simulation results.

As shown in the figure, at a lower gate voltage, the deviation between the model results from the experimental and the simulation result is larger, which can be reasoned as follows. The deviation between the threshold voltage model and the actual threshold voltage may result in inaccuracy in the drain current, which is a function of the gate overdrive voltage $\left(V_{\mathrm{GS}}-V_{T}\right)$. At a lower gate voltage, the effect of the deviation from the threshold voltage on the gate overdrive voltage becomes larger. Therefore, more deviation between the model and the data and simulation result could be seen at a lower gate voltage.

Fig. 4 shows (a) lattice temperature and (b) effective electron temperature versus drain voltage of the FD SOI NMOS device biased at $V_{\mathrm{GS}}=2 \mathrm{~V}$, with an LDD structure of $0.1 \mu \mathrm{m}$ in length doped with n-type densities of $2 \times 10^{18} \mathrm{~cm}^{-3}$ and $4 \times 10^{18} \mathrm{~cm}^{-3}$ based on the 2-D simulation and the model results. The lattice temperature results are based on (15) and (31). Using the electron temperature profile in the lateral channel [(8)], the effective electron temperature is defined as $T_{n \text { eff }}=$ $(1 /(L-\Delta L)) \int_{0}^{L-\Delta L} T_{n}(y) d y$. As shown in the figure, when the doping density of the LDD region is increased from $2 \times 10^{18}$ $\mathrm{cm}^{-3}$ to $4 \times 10^{18} \mathrm{~cm}^{-3}$, both the lattice and the effective electron temperatures increase in the device due to the reduced effectiveness of the LDD region. A more highly doped LDD region raises the electric field in the channel region, therefore both temperatures increase, which is especially serious at a high drain voltage. As shown in the figure, without considering energy transport (LT only), the lattice temperature may be overestimated, as shown in Fig. 4(a). In contrast, without considering self-heating (EB only), the effective electron temperature is under-estimated at a high drain voltage, as shown in Fig. 4(b).

Fig. 5 shows (a) lattice temperature and (b) effective electron temperature of the FD SOI NMOS device with and without an LDD structure of $0.1 \mu \mathrm{m}$ in length doped with an n-type density of $2 \times 10^{18} \mathrm{~cm}^{-3}$, biased at $V_{\mathrm{GS}}=2 \mathrm{~V}$, based on the model and the 2-D simulation results. As shown in Fig. 5(a), compared to the case without an LDD structure, the lattice temperature of the device with the LDD structure is lower, which is especially noticeable at a high drain voltage. At $V_{\mathrm{GS}}=2 \mathrm{~V}$, in the LDD 


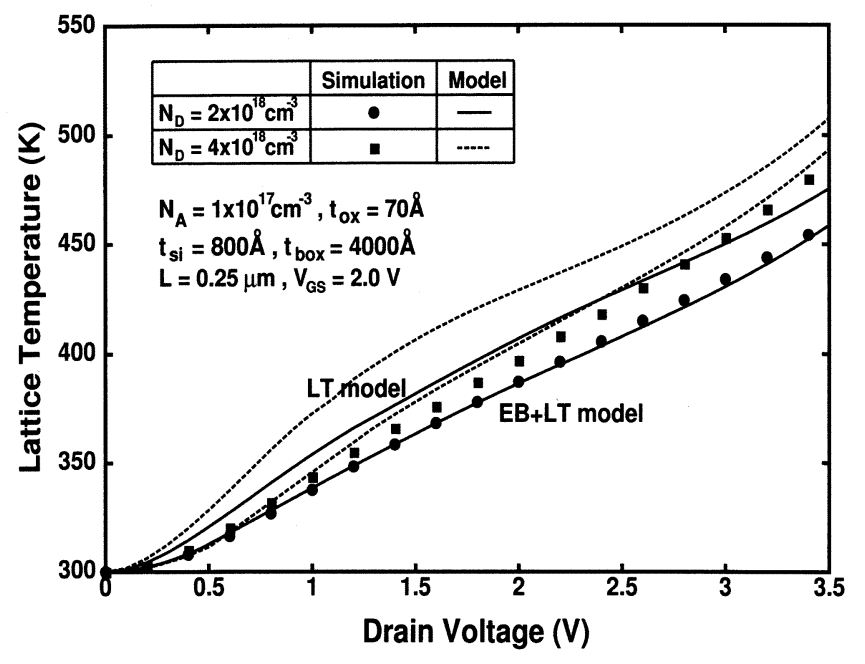

(a)

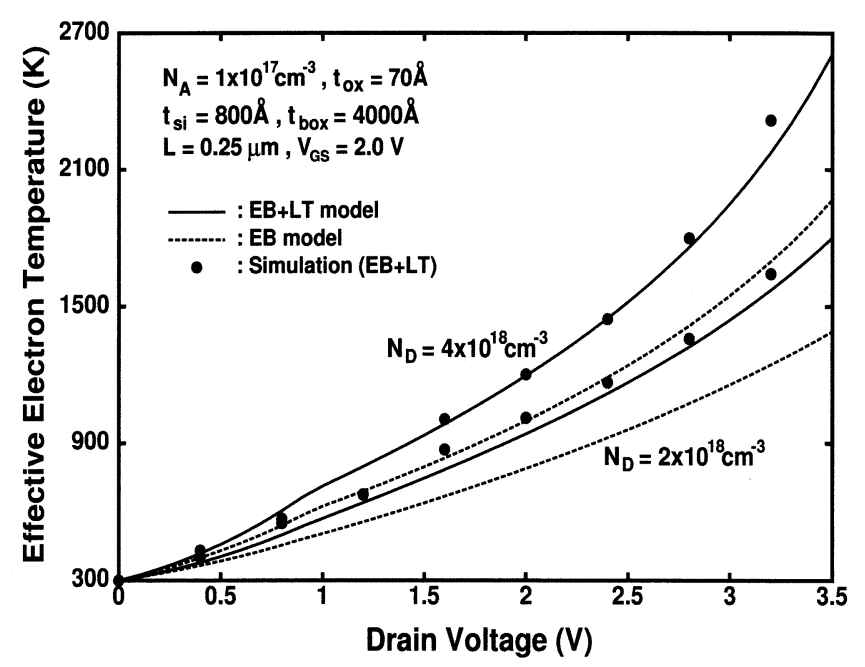

(b)

Fig. 4. (a) Lattice temperature and (b) effective electron temperature versus drain voltage of the FD SOI NMOS device with an LDD structure biased at $V_{\mathrm{GS}}=2 \mathrm{~V}$, based on the 2-D simulation and the model results.

device, the drain current is $0.33 \mathrm{~mA}$ (at $V_{\mathrm{DS}}=2 \mathrm{~V}$ ), which is $25 \%$ less as compare to the non-LDD device. With a smaller drain current, the lattice temperature is lower by $100 \mathrm{~K}$, due to a smaller power dissipation. The analytical model does predict the lattice temperature accurately as verified by the 2-D simulation results. As for the effective electron temperature shown in Fig. 5(b), the effective electron temperature is higher for both devices with and without the LDD structure. In addition, as verified by the 2-D simulation results, the effective electron temperature of the LDD one is smaller than that of the one without the LDD structure (non-LDD) due to a smaller electric field. For both non-LDD and LDD, the model provides an accurate prediction of the effective electron temperature as verified by the 2-D simulation results. From (11), the effective electron mobility is linearly proportional to the surface mobility $\left(\mu_{s}\left(T_{l}\right)\right)$, which is a function of the lattice temperature, and also inversely proportional to the difference between the electron and the lattice temperatures $\left(T_{n}-T_{l}\right)$. From (11), at a high drain voltage, the effective electron mobility is determined mainly by the effective

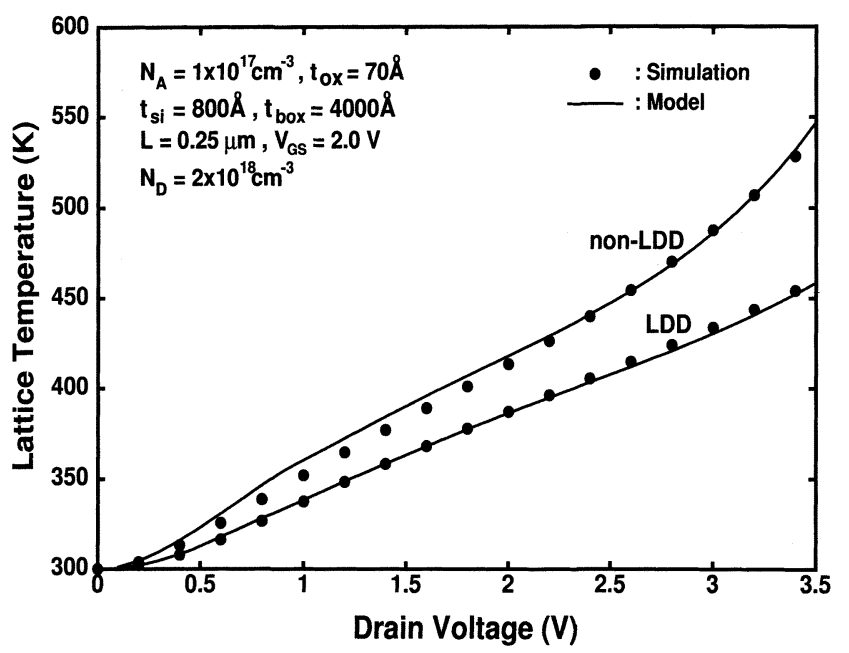

(a)

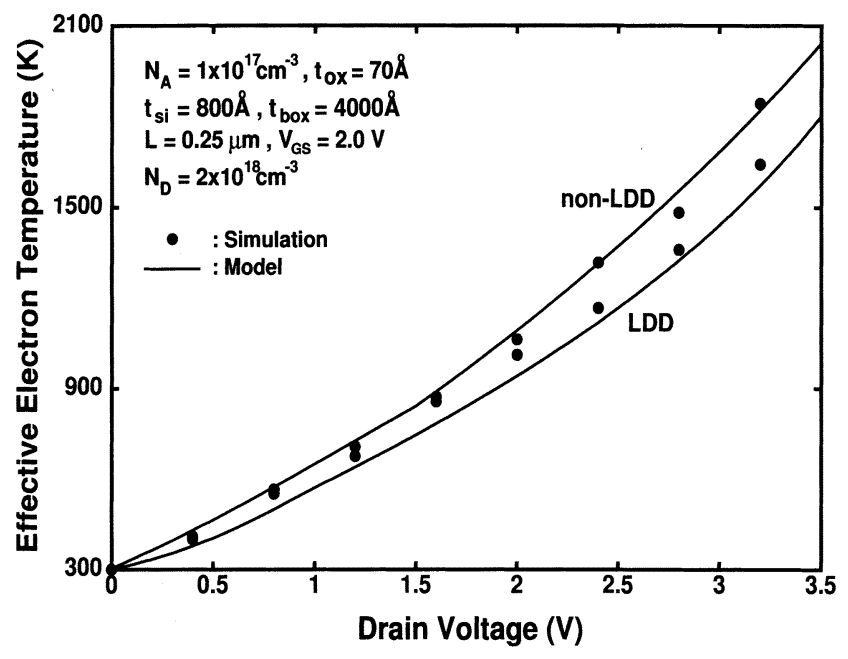

(b)

Fig. 5. (a) Lattice temperature, and (b) effective electron temperature versus drain voltage of the FD SOI NMOS device with and without an LDD structure, biased at $V_{\mathrm{GS}}=2 \mathrm{~V}$, based on the 2-D simulation and the model results.

electron temperature. On the other hand, at a low drain voltage, both electron and lattice temperatures are important in determining the mobility. The closed-form analytical model reported in this paper can be used for deep-submicron FD SOI NMOS devices with LDD structure with a channel length smaller than $0.25 \mu \mathrm{m}$. When the channel length becomes smaller, the electron/lattice temperature model becomes more important in determining the accuracy of a closed-form analytical model. With a shorter channel length, the electric field in the lateral channel increases. Therefore, the electron/lattice temperature model can facilitate a more accurate mobility model such that the drain current model can be more accurate.

\section{CONCLUSION}

In this paper, via an effective electron mobility model using the electron and the lattice temperatures based on the energy transport equation and the self-heating formula, a closed-form analytical drain current model for FD SOI NMOS devices with LDD structure has been reported. With a more heavily doped 
LDD region, the effective lattice and the electron temperatures in the FD SOI NMOS device are higher due to a higher electric field in the channel.

\section{APPENDIX}

In this Appendix, the derivation of the channel surface potential $[(5)]$ is presented. The derivation is divided into two regions: 1) the center channel region $(-L \leq y \leq 0)$ and 2) the source/drain-LDD region.

\section{A. Center Channel Region $(-L \leq y \leq 0)$}

For the 2-D Poisson's equation [(3)], with the boundary conditions and the approximation formula [(4)], one obtains the back surface potential

$$
\begin{aligned}
V_{\mathrm{sb}}(y)= & \frac{2 C_{s}+C_{\mathrm{ox}}}{2 C_{s}+C_{\mathrm{box}}} V_{\mathrm{sf}}(y)-\frac{C_{\mathrm{ox}}}{2 C_{s}+C_{\mathrm{box}}} V_{G} \\
& +\frac{C_{\mathrm{box}}}{2 C_{s}+C_{\mathrm{box}}} V_{\mathrm{SUB}} \\
a_{0}(y)= & V_{\mathrm{sf}}(y) \\
a_{1}(y)= & -\frac{\epsilon_{\mathrm{ox}}}{\epsilon_{\mathrm{si}}}\left(V_{G}-V_{\mathrm{sf}}(y)\right) \\
a_{2}(y)= & \frac{1}{2 t_{\mathrm{si}} \epsilon_{\mathrm{si}}}\left(C_{\mathrm{ox}}\left(V_{G}-V_{\mathrm{sf}}(y)\right)\right. \\
& \left.-C_{\mathrm{box}}\left(V_{\mathrm{sb}}(y)-V_{\mathrm{SUB}}\right)\right) .
\end{aligned}
$$

Using the approximation- $\left(\partial^{2} V_{(x, y)} / \partial y^{2}\right) \cong\left(1 / k_{s}\right)$ $\left(d^{2} V_{\mathrm{sf}}(y) / d y^{2}\right)$ and (3), one obtains

$$
\begin{aligned}
& \frac{d^{2} V_{\mathrm{sf}}(y)}{d y^{2}}-r_{0}^{2} V_{\mathrm{sf}}(y) \\
& =-\frac{q N k_{s}}{\epsilon_{\mathrm{si}}}+\beta_{1} \\
& r_{0}=\sqrt{\frac{2 k_{s}}{t_{\mathrm{si}} \epsilon_{\mathrm{si}}} \frac{C_{\mathrm{ox}} C_{\mathrm{box}}+C_{\mathrm{ox}} C_{s}+C_{\mathrm{box}} C_{s}}{2 C_{s}+C_{\mathrm{box}}}} \\
& \beta_{1}=-\frac{2 k_{s}}{t_{\mathrm{si}} \epsilon_{\mathrm{si}}} \frac{C_{\mathrm{ox}}\left(C_{s}+C_{\mathrm{box}}\right) V_{G}+C_{s} C_{\mathrm{box}} V_{\mathrm{SUB}}}{2 C_{s}+C_{\mathrm{box}}} .
\end{aligned}
$$

Solving (35) with $N=-N_{A}$, one obtains the potential and the electric field of the surface channel as

$$
\begin{aligned}
& V_{\mathrm{sf}}(y)=A_{0} e^{r_{0}(y+L)}+B_{0} e^{-r_{0}(y+L)}-\frac{q N_{A} k_{s}}{r_{0}^{2} \epsilon_{\mathrm{si}}}-\frac{\beta_{1}}{r_{0}^{2}} \\
& E_{\mathrm{sf}}(y)=-r_{0} A_{0} e^{r_{0}(y+L)}+r_{0} B_{0} e^{-r_{0}(y+L)} .
\end{aligned}
$$

At the ends of the channel region $(y=-L, y=0)$, from the above equation, one obtains

$$
\begin{aligned}
V_{\mathrm{sf}}(0) & =A_{0} e^{r_{0} L}+B_{0} e^{-r_{0} L}-\frac{q N_{A} k_{s}}{r_{0}^{2} \epsilon_{\mathrm{si}}}-\frac{\beta_{1}}{r_{0}^{2}} \\
E_{\mathrm{sf}}(0) & =-A_{0} r_{0} e^{r_{0} L}+B_{0} r_{0} e^{-r_{0} L} \\
V_{\mathrm{sf}}(-L) & =A_{0}+B_{0}-\frac{q N_{A} k_{s}}{r_{0}^{2} \epsilon_{\mathrm{si}}}-\frac{\beta_{1}}{r_{0}^{2}} \\
E_{\mathrm{sf}}(-L) & =-A_{0} r_{0}+B_{0} r_{0} .
\end{aligned}
$$

B. Source/Drain-LDD Region $\left(-L-L_{s} \leq y \leq-L, 0 \leq\right.$ $\left.y \leq L_{d}\right)$

In the source/drain-LDD region $\left(-L-L_{s} \leq y \leq-L, 0 \leq\right.$ $y \leq L_{d}$ ), the fringing electric field due to the oxide sidewall spacer is neglected, thus at the front gate interface the term $\left.(\partial V(x, y) / \partial x)\right|_{x=0}=0$. As a result, from (4) with the boundary conditions, the back surface potential is obtained

$$
\begin{aligned}
V_{\mathrm{sb}}(y) & =\frac{2 C_{s}}{2 C_{s}+C_{\mathrm{box}}} V_{\mathrm{sf}}(y)+\frac{C_{\mathrm{box}}}{2 C_{s}+C_{\mathrm{box}}} V_{\mathrm{SUB}} \\
a_{0}(y) & =V_{\mathrm{sf}}(y) \\
a_{1}(y) & =0 \\
a_{2}(y) & =\frac{1}{t_{\mathrm{si}}^{2}}\left(-\frac{C_{\mathrm{box}}}{2 C_{s}+C_{\mathrm{box}}} V_{\mathrm{sf}}(y)+\frac{C_{\mathrm{box}}}{2 C_{s}+C_{\mathrm{box}}} V_{\mathrm{SUB}} .\right.
\end{aligned}
$$

In addition, using 2-D Poisson's equation [(3)] with $N=N_{D}$, one obtains

$$
\begin{aligned}
\frac{d^{2} V_{\mathrm{sf}}(y)}{d y^{2}}-r_{1}^{2} V_{\mathrm{sf}}(y) & =-\frac{q N_{D} k_{s}}{\epsilon_{\mathrm{si}}}+\beta_{2} \\
r_{1} & =\sqrt{\frac{2 k_{s}}{t_{\mathrm{si}}^{2}} \frac{C_{\mathrm{box}}}{\left(2 C_{s}+C_{\mathrm{box}}\right)}} \\
\beta_{2} & =-\frac{2 k_{s}}{t_{\mathrm{si}}^{2}} \frac{C_{\mathrm{box}}}{2 C_{s}+C_{\mathrm{box}}} V_{\mathrm{SUB}} .
\end{aligned}
$$

Solving (41) in the source-LDD region $\left(-L-L_{s} \leq y \leq-L\right)$, the potential and electric field of the surface channel are

$$
\begin{aligned}
V_{\mathrm{sf}}(y)= & A_{1} e^{r_{1}\left(y+L+L_{s}\right)}+B_{1} e^{-r_{1}\left(y+L+L_{s}\right)} \\
& +\frac{q N_{D} k_{s}}{r_{1}^{2} \epsilon_{\mathrm{si}}}-\frac{\beta_{2}}{r_{1}^{2}} \\
E_{\mathrm{sf}}(y)= & -A_{1} r_{1} e^{r_{1}\left(y+L+L_{s}\right)}+r_{1} B_{1} e^{-r_{1}\left(y+L+L_{s}\right)} .
\end{aligned}
$$

At the two ends of the source-LDD region, namely, source ( $y=$ $\left.-L-L_{s}\right)$ and LDD/channel boundary $(y=-L)$, from the above equation, one obtains

$$
\begin{aligned}
V_{\mathrm{sf}}\left(-L-L_{s}\right) & =A_{1}+B_{1}+\frac{q N_{D} k_{s}}{r_{1}^{2} \epsilon_{\mathrm{si}}}-\frac{\beta_{2}}{r_{1}^{2}}=0 \\
V_{\mathrm{sf}}(-L) & =A_{1} e^{r_{1} L_{s}}+B_{1} e^{-r_{1} L_{s}}+\frac{q N_{D} k_{s}}{r_{1}^{2} \epsilon_{\mathrm{si}}}-\frac{\beta_{2}}{r_{1}^{2}} \\
E_{\mathrm{sf}}(-L) & =-A_{1} r_{1} e^{r_{1} L_{s}}+B_{1} r_{1} e^{-r_{1} L_{s}} .
\end{aligned}
$$

Similarly in the drain-LDD region $\left(0 \leq y \leq L_{d}\right)$, the potential and electric field of the surface channel are

$$
\begin{aligned}
& V_{\mathrm{sf}}(y)=A_{2} e^{r_{1} y}+B_{2} e^{-r_{1} y}+\frac{q N_{D} k_{s}}{r_{1}^{2} \epsilon_{\mathrm{si}}}-\frac{\beta_{2}}{r_{1}^{2}} \\
& E_{\mathrm{sf}}(y)=-A_{2} r_{1} e^{r_{1} y}+B_{2} r_{1} e^{-r_{1} y} .
\end{aligned}
$$

From the above equation, at the two ends of the drain-LDD region, namely, drain $\left(y=L_{d}\right)$ and LDD/channel boundary $(y=0)$, one obtains

$$
V_{\mathrm{sf}}\left(L_{d}\right)=A_{2} e^{r_{1} L_{d}}+B_{2} e^{-r_{1} L_{d}}+\frac{q N_{D} k_{s}}{r_{1}^{2} \epsilon_{\mathrm{si}}}-\frac{\beta_{2}}{r_{1}^{2}}=V_{D}
$$

$$
\begin{aligned}
& V_{\mathrm{sf}}(0)=A_{2}+B_{2}+\frac{q N_{D} k_{s}}{r_{1}^{2} \epsilon_{\mathrm{si}}}-\frac{\beta_{2}}{r_{1}^{2}} \\
& E_{\mathrm{sf}}(0)=-A_{2} r_{1}+B_{2} r_{1} .
\end{aligned}
$$


Since the potential and the electric field at the source/drain-LDD boundary points should be continuous, (38) should be equal to (49) and (39) should be equal to (45). As a result, one obtains

$$
\begin{aligned}
A_{1}= & \frac{1}{2} A_{0}\left(1+\frac{r_{0}}{r_{1}}\right) e^{-r_{1} L_{s}}+\frac{1}{2} B_{0}\left(1-\frac{r_{0}}{r_{1}}\right) e^{-r_{1} L_{s}} \\
& +\frac{1}{2} W_{0} e^{-r_{1} L_{s}} \\
B_{1}= & \frac{1}{2} A_{0}\left(1-\frac{r_{0}}{r_{1}}\right) e^{r_{1} L_{s}}+\frac{1}{2} B_{0}\left(1+\frac{r_{0}}{r_{1}}\right) e^{r_{1} L_{s}} \\
& +\frac{1}{2} W_{0} e^{r_{1} L_{s}} \\
A_{2}= & \frac{1}{2} A_{0}\left(1+\frac{r_{0}}{r_{1}}\right) e^{r_{0} L}+\frac{1}{2} B_{0}\left(1-\frac{r_{0}}{r_{1}}\right) e^{-r_{0} L} \\
& +\frac{1}{2} W_{0} \\
B_{2}= & \frac{1}{2}\left(1-\frac{r_{0}}{r_{1}}\right) A_{0} e^{r_{0} L}+\frac{1}{2}\left(1+\frac{r_{0}}{r_{1}}\right) B_{0} e^{-r_{0} L} \\
& +\frac{1}{2} W_{0} .
\end{aligned}
$$

From (50)-(53) and (44) and (48), one obtains (5).

\section{ACKNOWLEDGMENT}

The authors would like to thank the reviewers for their thorough help in reviewing this paper such that the quality of it could have been improved.

\section{REFERENCES}

[1] J. B. Kuo and S. C. Lin, Low-Voltage SOI CMOS VLSI Devices and Circuits. New York: Wiley, 2001. ISBN 0471417777.

[2] J. B. Kuo and K. W. Su, CMOS VLSI Engineering: Silicon on Insulator (SOI). Norwell, MA: Kluwer, 1998. ISBN 0792382722.

[3] K. Imai, H. Onishi, K. Yamaguchi, K. Inoue, Y. Matsubara, A. Ono, and T. Horiuchi, "A $0.8 \mu \mathrm{m}$ fully-depleted CMOS on 30-nm thick SOI for sub-0.1-V operation," in Symp. VLSI Tech. Dig., 1998, pp. 116-117.

[4] E. P. Ver Ploeg, C. T. Nguyen, S. S. Wong, and J. D. Plummer, "Parasitic bipolar gain in fully-depleted $n$-channel SOI MOSFETs," IEEE Trans. Electron Devices, vol. 41, pp. 970-977, June 1994.

[5] J. Jomaah, F. Balestra, and G. Ghibaudo, "Self-heating effect in SOI MOSFETs operated at low temperature," in SOI Conf. Dig., 1993, pp. $82-83$.

[6] J. Jomaah, G. Ghibaudo, F. Balestra, and J. L. Pelloie, "Impact of selfheating effect on the design of SOI devices versus temperature," in SOI Conf. Dig., 1995, pp. 114-115.

[7] Y.-G. Chen, S.-Y. Ma, J. B. Kuo, Z. Yu, and R. W. Dutton, "An analytical drain current model considering both electron and lattice temperatures simultaneously for deep submicron ultrathin SOI NMOS devices with self-heating," IEEE Trans. Electron Devices, vol. 42, pp. 899-906, May 1995.

[8] K. W. Su and J. B. Kuo, "A nonlocal impact ionization/lattice temperature model for VLSI double-gate ultrathin SOI NMOS devices," IEEE Trans. Electron Devices, vol. 44, pp. 324-330, Feb. 1997.

[9] Y. Omura, "Physical background of hot-carrier-induced abnormal $g_{m}$ degradation in a sub-0.1- $\mu$ m-channel nMOSFETs/SIMOX with an LDD structure," in SOI Conf. Dig., 1997, pp. 96-97.

[10] F. L. Duan, S. P. Sinha, D. E. Ioannou, and F. T. Brady, "LDD design tradeoffs for single transistor latch-up and hot carrier degradation control in accumulation mode FD SOI MOSFETs," IEEE Trans. Electron Devices, vol. 44, pp. 972-977, June 1997.

[11] J. Chen, S. Parke, J. King, F. Assaderaghi, P. K. Ko, and C. Hu, "A high speed SOI technology with $12 \mathrm{ps} / 18 \mathrm{ps}$ gate delay operating at $5 \mathrm{~V} / 1.5$ V," in IEDM Tech. Dig., 1992, pp. 35-38.
[12] Y. Yamaguchi, T. Iwamatsu, H.-O. Joachim, H. Oda, Y. Inoue, T. Nishimura, and K. Tsukamoto, "Source-to-drain breakdown voltage improvement in ultra-thin-film SOI MOSFETs using a gate-overlapped LDD structure," IEEE Trans. Electron Devices, vol. 41, pp. 1222-1226, July 1994.

[13] A. S. Nicolett, J. A. Martino, E. Simoen, and C. Claeys, "A new method to extract the LDD doping concentration on fully-depleted SOI nMOSFET at 300 K," Dev., Circ., Syst., pp. D45/1-D45/5, 2000.

[14] S.-C. Lin and J. B. Kuo, The fringing electric field effect on the shortchannel effect threshold voltage of fully-depleted (FD) SOI NMOS devices with the lightly-doped drain (LDD)/sidewall oxide spacer structure, in IEEE Trans. Electron Devices, to be published.

[15] S. S. Chen and J. B. Kuo, "Deep submicrometer double-gate fully-depleted SOI PMOS devices: A concise short-channel effect threshold voltage model using a quasi-2-D approach," IEEE Trans. Electron Devices, vol. 43, pp. 1387-1393, Sept. 1996.

[16] Y. Cheng and T. A. Fjeldly, "Unified physical $I-V$ model including self-heating effect for fully-depleted SOI/MOSFETs," IEEE Trans. Electron Devices, vol. 43, pp. 1291-1296, Aug. 1996.

[17] N. Yasuda, S. Ueno, K. Taniguchi, C. Hamaguchi, Y. Yamaguchi, and T. Nishimura, "Analytical device model of SOI MOSFETs including self-heating effect," Jpn. J. Appl. Phys., vol. 30, pp. 3677-3684, Dec. 1991.

[18] Y. A. El-Mansy and A. R. Boothroyd, "A simple two-dimensional model for IGFET operation in the saturation region," IEEE Trans. Electron Devices, vol. ED-34, pp. 254-262, Mar. 1987.

[19] K. Mayaram, J. C. Lee, and C. Hu, "A model for the electric field in lightly doped drain structures," IEEE Trans. Electron Devices, vol. ED-34, pp. 1509-1518, July 1987.

[20] Device simulation software, 1998. ATLAS.

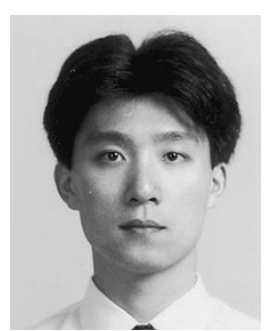

Shih-Chia Lin was born in Taichung, Taiwan, R.O.C., on October 27, 1970. He received the B.S. and $\mathrm{Ph} . \mathrm{D}$. degrees in electrical engineering from National Taiwan University, Taipei, in 1992 and 2001, respectively. His Ph.D. dissertation was entitled "Compact modeling of bulk and SOI CMOS VLSI devices."

Since 2001, he has been a Research Assistant Professor in the Department of Electrical and Computer Engineering, University of Waterloo, Waterloo, ON, Canada. His research expertise is in the field of compact modeling of CMOS VLSI devices.

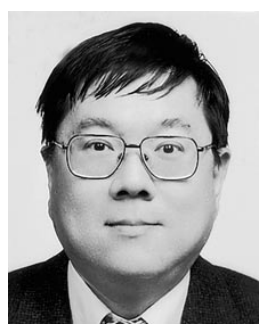

James B. Kuo (S'85-M'85-SM'92-F'00) received the B.S.E.E. degree from National Taiwan University (NTU), Taipei, Taiwan, R.O.C., in 1977, the M.S.E.E. degree from The Ohio State University, Columbus, in 1978, and the Ph.D.E.E. degree from Stanford University, Stanford, CA, in 1985.

He was with Penril Data Communications and Racal Vadic (1978-1981) as a Research Engineer working on integrating telecommunication modem chips using CMOS technology. From 1985 to 1987, he was an Engineering Research Associate in the IC Lab of Stanford University, working on BiCMOS devices. In 1987, he joined NTU as an Associate Professor and, since 1990, he has been a Professor at the same university. In 2000, he joined the University of Waterloo, Waterloo, ON, Canada, as a tenured Full Professor, on leave from the NTU. In 2001, he was awarded the prestigious Canada Research Chair Professor by the Canadian government. His research expertise is in the field of low-voltage CMOS VLSI circuits and SPICE compact modeling of deep-submicrometer bulk and SOI CMOS and BiCMOS VLSI devices. He has published 250 technical papers including 110 international journal papers, of which 50 are IEEE journal papers. He holds 16 invention patents including seven U.S. patents on low-voltage CMOS VLSI circuits. He has also authored nine books.

Dr. Kuo is an IEEE Fellow for contributions to modeling CMOS VLSI devices. He serves as an international advisory committee member for the IEEE Circuits and Devices Magazine and the membership committee chair for the IEEE Electron Devices Society. He is also an IEEE Electron Devices Society distinguished lecturer. As a technical leader, he has graduated 50 M.S. and Ph.D. students specialized in CMOS circuit designs and device modeling, currently working in leading U.S. and Taiwan microelectronics companies. 